\title{
Effect of supersymmetric phases on lepton dipole moments and rare lepton decays
}

\author{
Werner Porod* \\ Institut für Theoretische Physik, Universität Zürich, CH-8057 Zürich, Switzerland \\ E-mail: iporod@physik.unizh.ch'
}

\begin{abstract}
We study the effect of SUSY phases on rare decays of leptons and on their magnetic and electric dipole moments. We focus on two scenarios: (i) selectron - stau mixing and (ii) general three generation mixing. In both cases we consider the most general mass matrices for sleptons within the MSSM including left-right mixing, flavour mixing and complex phases. We emonstrate that contrary to common belief the phase of $\mu$ can be large even for slepton masses as small as $200 \mathrm{GeV}$ provided the lepton flavour violating parameters are complex.
\end{abstract}

\section{Introduction}

The results of the recent neutrino experiments [i] are a clear indication for non-vanishing neutrino masses and violation of individual lepton numbers. For this reason one expects also flavour violating effects also for charged leptons. However, lepton flavour violation (LFV) of charged leptons is severely constrained by experiments: $B R(\mu \rightarrow e \gamma)<1.2 \cdot 10^{-11}$, $B R(\tau \rightarrow e \gamma)<2.7 \cdot 10^{-6}, B R(\tau \rightarrow \mu \gamma)<1.1 \cdot 10^{-6}[2]$. In analogy to quarks, lepton flavour violation may also be related to $\mathrm{CP}$ violation. The limits on leptonic $\mathrm{CP}$ violation, such as the bound of $10^{-27} \mathrm{ecm}$ on the electric dipole moment of the electron, are also quite strong. Within the standard model (SM) framework this is somewhat less significant because the leptonic dipole moments, being a three-loop effect, are generically small [3페]

Supersymmetric (SUSY) extensions of the SM contain additional sources for LFV and $\mathrm{CP}$ violation in the slepton sector and the corresponding effects can be generically large. Moreover, there are additional sources for $\mathrm{CP}$ violation in the chargino and neutralino sector. Consequently, rare processes and $C P$ violation impose significant bounds on the flavour violating terms in the slepton mass matrices. Various phenomenological implications of LFV with real mass matrices for sleptons were extensively studied in the literature

\footnotetext{
${ }^{*}$ Speaker.
} 
[i] tion and decays of supersymmetric particles despite the stringent experimental bounds on flavour violating lepton decays. On the other hand, studies with complex parameters have

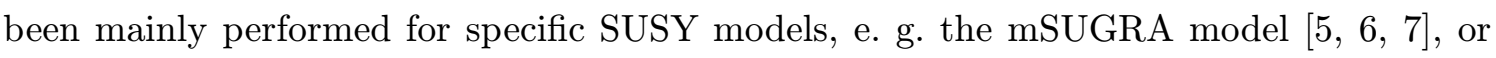

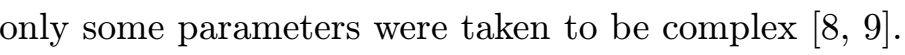

Here we study lepton flavour violation and $\mathrm{CP}$ violation in the lepton sector in the general situation, where all parameters can be complex, in particular the LFV entries of the slepton mass matrices. This important generalization is quite natural and is motivated by the close analogy between quarks and leptons and their supersymmetric partners. The phase of the $C K M$ matrix is large and the the smallness of certain $C P$-violating observables (in the K-system) is due to the structure of the theory.

The present experiments impose rather stringent bounds on SUSY phases because CP-violating effects such as electric dipole moments can be quite large in SUSY. Therefore it is often suggested that the phases are small altogether [i $10 \overline{0}]$. Since this view is in a sense contradictory to the large phase of the standard model, it is desirable to carry out a general study of flavour and CP violation with complex parameters in order to see whether the restrictions can be softened. Furthermore, large leptonic $C P$ violation together with leptogenesis [1] ${ }_{1}^{1} \mathbf{1}_{1}$ may also be the key to the baryon asymmetry of the universe. One goal of our work therefore is to determine whether large phases are indeed possible and not in contradiction with experiment.

General models with soft SUSY breaking terms contain a large number of complex parameters. Consequently, each observable can have contributions from several parameters and no clear statements on their allowed ranges may be possible. As a second goal of our study, we want to show that, nevertheless, important results can be obtained because the present limits on rare processes in the lepton sector are so strong. Furthermore, several experiments with substantially increased sensitivity are planned for the near future and will lead to even more decisive information. Future measurements of $d_{e}[1 \overline{2} \overline{2}]$ and $d_{\mu}[1 \overline{3} \overline{3}$ may substantially improve the sensitivity to $10^{-29}$ and $10^{-24}$, respectively. New experiments for the search of the rare decay $\mu \rightarrow e \gamma$ at the level of $10^{-14}\left[\begin{array}{l}\overline{1} \\ \overline{4}\end{array}\right]$ are also underway.

As there are many parameters involved, we will fix the modulus of the flavour conserving parameters and vary the flavour violating parameters as well as all possible phases. We have checked that the qualitative features of this study do not change if one varies the flavour conserving parameters. The processes we will study are the rare leptonic decays $\mu \rightarrow e \gamma, \tau \rightarrow e \gamma$ and $\tau \rightarrow \mu \gamma$ and the electric (EDM) and magnetic dipole moments (MDM) of $e, \mu$ and $\tau$. We will use the present experimental bounds, $d_{e}<1.5 \cdot 10^{-27}$

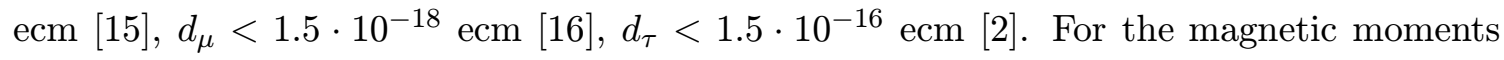
we assume that the supersymmetric contribution is limited by the experimental errors of $\pm 10^{-12}$ and \pm 0.058 for $a_{e}$ and $a_{\tau}$ respectively. For the muon, there are new measurements

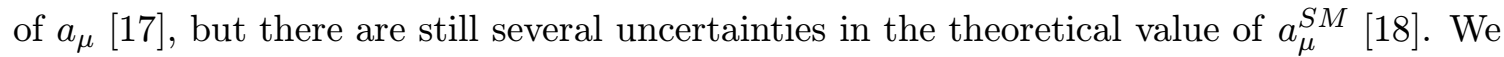
will take the conservative range $a_{\mu}^{e x p}-a_{\mu}^{S M}=43 \cdot 10^{-10}$, which corresponds to the largest deviation in the calculations.

The organization of the paper is as follows: In the next section we define the parameters and fix the notation. In section 3 we study a scenario with complex parameters but 
without flavour violation. In section 4 the general situation with lepton flavour violation and complex parameters is studied. The conclusions are drawn in section 5 .

\section{The basic parameters}

The model under study is the general Minimal Supersymmetric Standard Model (MSSM). The parameters relevant for this study are the lepton Yukawa coupling $Y^{E}$ and higgsino mixing parameter $\mu$ of the superpotential as well as the following part of the soft SUSY breaking Lagrangian:

$$
\mathcal{L}=M_{L, i j}^{2} \tilde{l}_{L, i} \tilde{l}_{L, j}^{*}+M_{E, i j}^{2} \tilde{l}_{R, i} \tilde{l}_{R, j}^{*}+\left(A_{i j} H_{1} \tilde{l}_{L, i} \tilde{l}_{R, j}^{*}+M_{1} \tilde{b} \tilde{b}+M_{2} \tilde{w}^{a} \tilde{w}^{a}+h . c .\right)
$$

$M_{1}$ and $M_{2}$ are the $U(1)$ and $S U(2)$ gaugino mass parameters, respectively. $M_{L}^{2}$ and $M_{E}^{2}$ are the soft SUSY breaking mass matrices for left and right sleptons, respectively, and the $A_{i j}$ are the trilinear soft SUSY breaking couplings of the sleptons and Higgs boson. $M_{1}$, $M_{2}, M_{L, i j}^{2}=\left(M_{L, j i}^{2}\right)^{*}, M_{E, i j}^{2}=\left(M_{E, i j}^{2}\right)^{*}$ and $A_{i j}$ are complex; note that $A_{i j} \neq A_{j i}^{*}$ for $i \neq j$. The most general charged slepton mass matrix including left-right mixing as well as flavor mixing is usually written in the form

$$
M_{\tilde{l}}^{2}=\left(\begin{array}{cc}
M_{L L}^{2} & M_{L R}^{2 \dagger} \\
M_{L R}^{2} & M_{R R}^{2}
\end{array}\right),
$$

where the entries are $3 \times 3$ matrices. In terms of the parameters introduced in $\left(\overline{2} . \overline{2}_{1}^{\prime}\right)$, they are given by

$$
\begin{aligned}
& M_{L L, i j}^{2}=M_{L, i j}^{2}+\frac{v_{d}^{2} Y_{k i}^{E *} Y_{k j}^{E}}{2}+\frac{\left(g^{\prime 2}-g^{2}\right)\left(v_{d}^{2}-v_{u}^{2}\right) \delta_{i j}}{8}, \\
& M_{L R, i j}^{2}=\frac{v_{d} A_{i j}^{*}-\mu v_{u} Y_{i j}^{E}}{\sqrt{2}}, \\
& M_{R R, i j}^{2}=M_{E, i j}^{2}+\frac{v_{d}^{2} Y_{i k}^{E} Y_{j k}^{E *}}{2}-\frac{g^{\prime 2}\left(v_{d}^{2}-v_{u}^{2}\right) \delta_{i j}}{4} .
\end{aligned}
$$

The indices $i, j, k=1,2,3$ characterize the flavors $e, \mu, \tau . \quad v_{u}$ and $v_{d}$ are the vacuum expectation values of the neutral Higgs fields (with $\tan \beta=v_{u} / v_{d}$ ). In what follows we will work in a basis where $M_{2}$ is real and where the lepton Yukawa coupling is real and flavour diagonal. Both assumptions can be done without loss of generality, because (i) only phase differences matter and (ii) there are no right-handed neutrinos in the low energy spectrum. Similarly, one finds for the sneutrinos

$$
M_{\tilde{\nu}, i j}^{2}=M_{L, i j}^{2}+\frac{\left(g^{2}+g^{\prime 2}\right)\left(v_{d}^{2}-v_{u}^{2}\right) \delta_{i j}}{8} .
$$

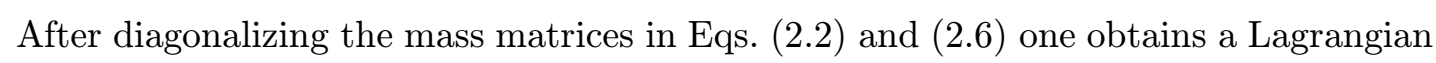
which contains complex lepton flavour violating couplings. The corresponding formulas are given in ref. [1] $\left.{ }_{1}^{1} \overline{1}\right]$. These couplings give, at the 1-loop level, contributions to the anomalous 

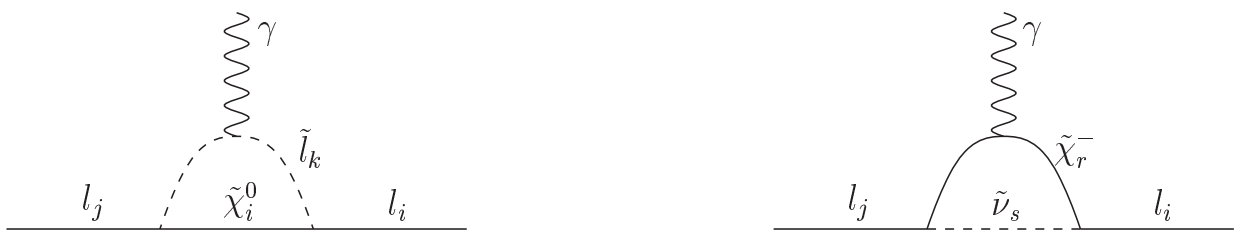

Figure 1: Generic diagrams contributing to $\Delta a_{l}, d_{l}, l_{j} \rightarrow l_{i} \gamma$.

magnetic moments of the leptons $a_{l}$, the electric dipole moments $d_{l}$ and to rare lepton decays such as $l_{j} \rightarrow l_{i} \gamma$. All these observables are induced by the same type of amplitude

$$
T=i e \epsilon^{\mu *} \frac{q^{\nu}}{2 m_{l_{j}}} \bar{l}_{i} \sigma_{\mu \nu}\left(a_{i j}^{L} P_{L}+a_{i j}^{R} P_{R}\right) l_{j}
$$

arising from the diagrams shown in Fig. 1 . Here we take $i \leq j$. The formulas for the coefficients $a_{i j}^{L}$ and $a_{i j}^{R}$ can be found in ref. [i [i $\left.g_{1}^{1}\right]$. The observables $\Delta a_{i}, d_{i}$ and $l_{j} \rightarrow l_{i} \gamma$ can be expressed as:

$$
\begin{aligned}
\Delta a_{i} & =\frac{1}{2} \operatorname{Re}\left(a_{i i}^{L}+a_{i i}^{R}\right) \\
\frac{1}{e} d_{i} & =\frac{1}{2} \operatorname{Im}\left(-a_{i i}^{L}+a_{i i}^{R}\right) \\
\Gamma\left(l_{j} \rightarrow l_{i} \gamma\right) & =\frac{\alpha m_{l, j}}{16}\left(\left|a_{i j}^{L}\right|^{2}+\left|a_{i j}^{R}\right|^{2}\right)
\end{aligned}
$$

where in the last equation we have neglected terms of order $\mathrm{O}\left(m_{l_{i}} / m_{l_{j}}\right)$.

\section{The flavour conserving case}

We begin our investigation with a point which is close to the SPS\#1a point [2] $\left.\overline{2}_{-1}^{0}\right]: M_{L, 11}^{2}=$ $M_{L, 22}^{2}=202.3^{2} \mathrm{GeV}^{2}, M_{L, 33}^{2}=201.5^{2} \mathrm{GeV}^{2}, M_{E, 11}^{2}=M_{E, 22}^{2}=138.7^{2} \mathrm{GeV}^{2}, M_{E, 33}^{2}=$ $136.3^{2} \mathrm{GeV}^{2}, A_{11}=-7.567 \cdot 10^{-3} \mathrm{GeV}, A_{22}=-1.565 \mathrm{GeV}, A_{33}=-26.326 \mathrm{GeV}, M_{1}=$ $107.9 \mathrm{GeV}, M_{2}=208.4 \mathrm{GeV}, \mu=365 \mathrm{GeV}, \tan \beta=10$. Note that the $A$ parameters are already multiplied by the lepton Yukawa couplings. We obtain the following SUSY contributions to the observables: $d_{e}=d_{\mu}=d_{\tau}=0, \Delta a_{e}=6.8 \cdot 10^{-14}, \Delta a_{\mu}=2.9 \cdot 10^{-9}$, $\Delta a_{\tau}=8.4 \cdot 10^{-7}$.

In the flavour conserving case the electron EDM constrained the phase $\varphi_{\mu}$ of the

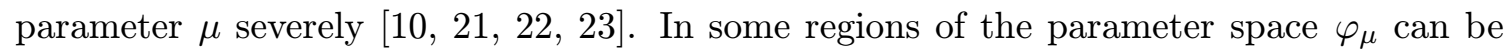
about $\pi / 10$ for slepton masses as light as $\mathrm{O}(200) \mathrm{GeV}$ provided there are cancellations between the chargino and neutralino contributions [202 2$]$. This is due to an interplay of the phases $\varphi_{A_{11}}$ and $\varphi_{U_{1}}$, where $\varphi_{U_{1}}$ is the phase of the $M_{1}$ parameter. In Fig. 2 a we show the range of the $\varphi_{\mu}-\varphi_{U_{1}}$ plane allowed by the electron EDM; $\varphi_{A_{11}}$ is varied in the full range. The two bands collapse to lines for fixed $\varphi_{A_{11}}$. Similar results have also been found in [2] $\left.\overline{2}^{2} \bar{i}\right]$. In Fig. 2 b the SUSY contribution $\Delta a_{\mu}$ to the anomalous magnetic moment of the muon is shown. The two bands correspond to the cases $\varphi_{\mu} \simeq 0$ and $\varphi_{\mu} \simeq \pi$. We see that while the EDM leaves a twofold ambiguity for the phase $\varphi_{\mu}$, the $C P$-conserving anomalous magnetic 

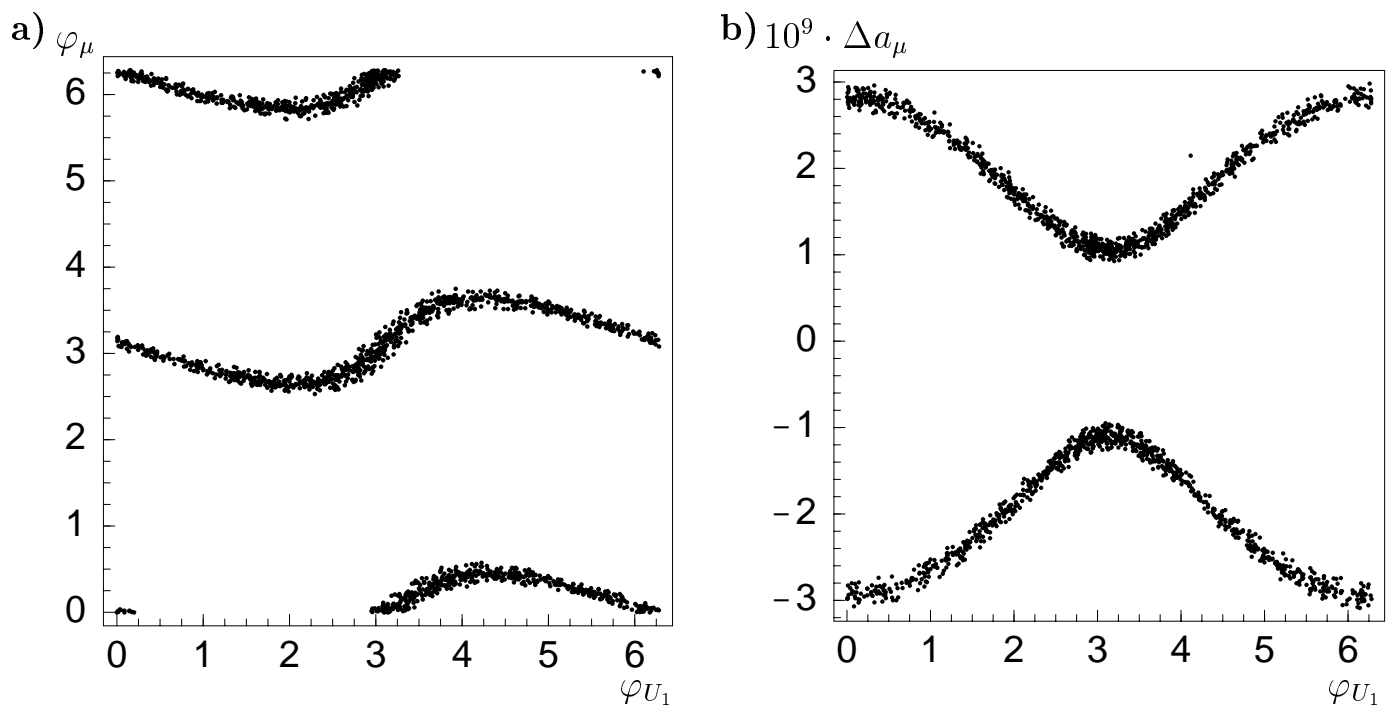

Figure 2: a) Allowed regions in the $\varphi_{\mu}-\varphi_{U_{1}}$ plane by the electron EDM; b) SUSY contribution $\Delta a_{\mu}$ to the anomalous magnetic moment of the muon.

moment discriminates between the two values - indeed the lower band is already excluded and $\varphi_{\mu}$ must be near $\pi$ as has been also observed in ref. [2] $\left.\overline{2}_{-1}\right]$. This clearly shows that phases are also important for CP-conserving observables and that a combined analysis of all effects is necessary.

Despite the new freedom, $\mu$ is still basically real. Unless the $A$ parameters are substantially larger, this conclusion remains. However, bigger values of $\left|A_{11}\right|$ are in contradiction with stability arguments for the potential. As we will see, the complex phases of flavour violating parameters change this picture.

\section{Including flavour violation}

In this section we consider two scenarios. First we study the case of $\tilde{e}-\tilde{\tau}$ mixing and in a second step the general three generation case. Details for $\tilde{e}-\tilde{\mu}$ mixing as well as for $\tilde{\mu}-\tilde{\tau}$ mixing can be found in ref. [i] $\underline{1} \overline{9}]$.

\section{$4.1 \tilde{e}-\tilde{\tau}$ mixing}

Starting from our reference point, we add the following flavour violating terms: $M_{L, 13}^{2}=$ $1500 \mathrm{GeV}^{2}, M_{E, 13}^{2}=2000 \mathrm{GeV}^{2}, A_{31}=A_{13}=20 \mathrm{GeV}$ yielding $\mathrm{BR}(\tau \rightarrow e \gamma)=1.05 \cdot 10^{-6}$. The effect of the phases is shown in Fig. $\underline{1}_{\mathbf{1}}$. Each individual contribution from the various phases $\varphi_{M_{E, 13}^{2}}, \varphi_{M_{L, 13}^{2}}, \varphi_{A_{13}}$ and $\varphi_{A_{31}}$ is similar in size to that of $\varphi_{\mu}$. If only one of these phases would generate the electron EDM, it would have to be very near zero or $\pi$ because the effect is of order $10^{-23} \mathrm{ecm}$ as seen in the figure. But if there are several contributions, the phases can be arbitrarily large, since various contributions can cancel each other. Such a cancellation is not obvious, because the bounds on $\Delta a_{e}$ and $\operatorname{BR}(\tau \rightarrow e \gamma)$ must be satisfied and the parameters are already constrained. 
a) $10^{23} \cdot d_{e}$

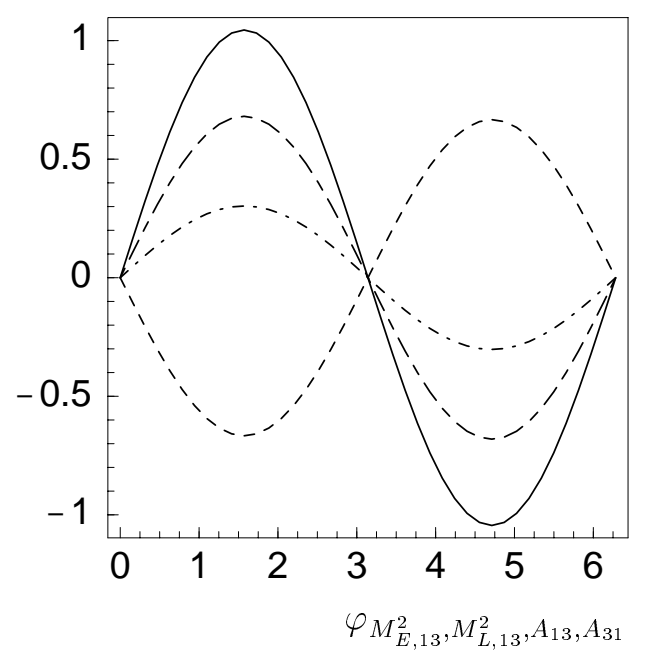

b) $10^{13} \cdot \Delta a_{e}$

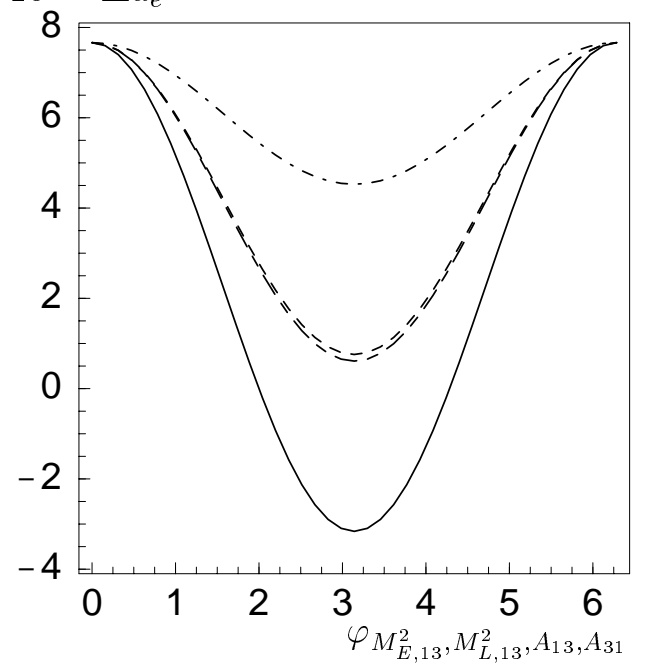

Figure 3: a) $d_{e}$ and b) SUSY contributions to $a_{e}$ as a function of $\varphi_{M_{E, 13}^{2}}$ (full line), $\varphi_{M_{L, 13}^{2}}$ (dashed line), $\varphi_{A_{13}^{l}}$ (dashed dotted line) and $\varphi_{A_{31}}$ (long-short dashed line) for $\varphi_{\mu}=0$.
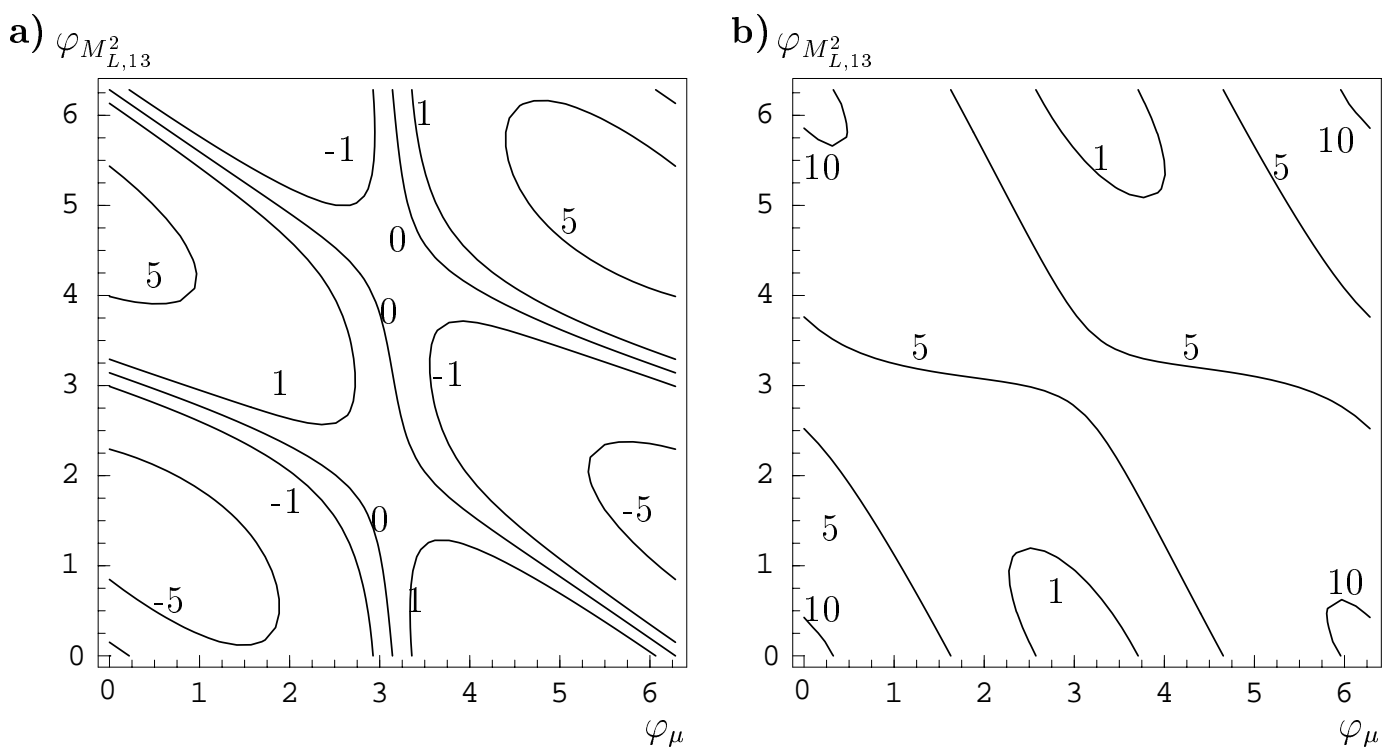

Figure 4: a) $10^{24} d_{e}$ and b) $10^{7} \mathrm{BR}(\tau \rightarrow e \gamma)$ in the $\varphi_{\mu}-\varphi_{M_{L, 13}^{2}}$ plane.

In Fig. 运 the contour plot for $d_{e}$ as a function of $\varphi_{\mu}$ and $\varphi_{M_{L, 13}^{2}}$ is shown. Very small $d_{e}$ consistent with the experimental upper bound can be obtained for all (!) values of $\varphi_{\mu}$ provided that also the phase $\varphi_{M_{L, 13}^{2}}$ of the flavour violating parameter $M_{L, 13}^{2}$ is large. There are roughly two allowed regions. In one region, the two phases are equal and opposite and there is a cancellation between the lepton flavour conserving and the lepton flavour violating contributions. In this case, the phase of $\mu$ can be large indeed. In the other region, $\varphi_{\mu}$ is around $\pi$ and there is only a weak dependence on $\varphi_{M_{L, 13}^{2}}$. In this situation, the contribution from $M_{L, 13}^{2}$ is not important for the dipole moment. Note that here only 
the phases shown in the plot are varied, while the others are set equal to 0. Similar results are obtained in the $\varphi_{\mu}-\varphi_{M_{E, 13}^{2}}$ plane and in the $\varphi_{\mu}-\varphi_{A_{31}}$ plane [i] $\left.\underline{1}_{1}\right]$.

As can be seen from Fig. Aiph, the decay rate for $\tau \rightarrow e \gamma$ varies within an order of magnitude over the plot. However, if further experiments would establish a considerably lower limit or measure the branching ratio with $50 \%$ or better, the phases could be severely constrained. This underlines clearly the strength of a combined analysis, once the basic supersymmetric parameters are known.

\subsection{The three generation case}

We now study the most general mixing in the slepton sector. We take our reference point and add all possible phases and generation mixing terms. The moduli of the off-diagonal terms vary between zero and the following upper bounds: $\left|M_{E, 12}^{2}\right|,\left|M_{L, 12}^{2}\right| \leq 10 \mathrm{GeV}^{2}$, $\left|M_{E, 13}^{2}\right|,\left|M_{E, 23}^{2}\right|,\left|M_{L, 13}^{2}\right|,\left|M_{L, 23}^{2}\right| \leq 1000 \mathrm{GeV}^{2},\left|A_{12}\right|,\left|A_{21}\right| \leq 0.05 \mathrm{GeV},\left|A_{13}\right|,\left|A_{31}\right|,\left|A_{23}\right|$, $\left|A_{32}\right| \leq 20 \mathrm{GeV}$. All phases are varied in the range between 0 and $2 \pi$.

In Fig. constraints from the EDMs and the rare lepton decays. Comparing Fig. ${ }_{-1}^{1}$ with Fig. ${ }_{-1}^{2}$ one sees that maximal values for both $\varphi_{\mu}$ and $\varphi_{U_{1}}$ are possible. This is due to cancellations between the lepton flavour conserving and the lepton flavour violating contributions. Note that such cancellations are possible even for slepton masses as small as $200 \mathrm{GeV}$. In Fig. we show the SUSY contribution $\Delta a_{\mu}$ to the anomalous magnetic moment of the muon as a function of $\varphi_{U_{1}}$, varying all parameters and phases in the range given above and fulfilling the constraints from the EDMs and the rare lepton decays.

Lepton flavour violation leads to the violation of the naive scaling relations like $d_{e} / d_{\mu} \simeq$ $m_{e} / m_{\mu}[$ [i] In Fig. ' $6 \overline{1}=1$ we show the results for $\Delta a_{e}$ versus $\Delta a_{\mu}$ and $d_{e}$ versus $d_{\mu}$. One sees that the naive relation $\Delta a_{e} / \Delta a_{\mu} \propto\left(m_{e} / m_{\mu}\right)^{2}$ is largely maintained after imposing the experimental constraints arising from EDMs and rare decays even if one allows for the most general flavour structure. However, there are parameter points where the simple $\Delta a_{\mu}-\Delta a_{e}$ scaling

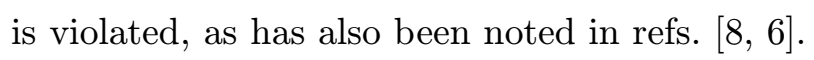

The situation changes completely for the electric dipole moments where the correlation between $d_{e}$ and $d_{\mu}$ is completely destroyed once all possible flavour violating parameters are taken into account. The reason for the difference between EDMs and the MDMs is that in the case of the $d_{e}$ cancellations of at least of two orders of magnitude are required to satisfy the experimental bounds implying that $d_{e}$ is no longer proportional to $m_{e}$. We have checked that in the case, where a larger modulus of $d_{e}$ is allowed, the proportionality to $m_{e}$ is restored except for the region around 0. Moreover, we have checked that the ratio $d_{\mu} / d_{\tau}$ is still proportional to $m_{\mu} / m_{\tau}$.

Fig. $\bar{t}_{1}$ shows the allowed regions for the complex parameters $M_{E, 13}^{2}$ and $A_{13}$ for $\varphi_{\mu}=$ $\pi / 2$. All other phases have been varied in the range $(0,2 \pi)$. Similar results are obtained for the parameters $M_{L, 13}^{2}$ and $A_{31}\left[{ }_{1}^{1} \overline{9}_{1}^{i}\right]$. Again, the allowed regions are large. Note, that the $\left|M_{E, 13}^{2}\right|,\left|M_{L, 13}^{2}\right|$ can go up to $5 \%$ of $\left|M_{E, 33}^{2}\right|$ and $\left|M_{L, 33}^{2}\right|$, respectively. $\left|A_{13}\right|$ and $\left|A_{31}\right|$ can have the same order of magnitudes as $\left|A_{33}\right|$. In case of $\varphi_{\mu}=0$ roughly the same areas 
a)

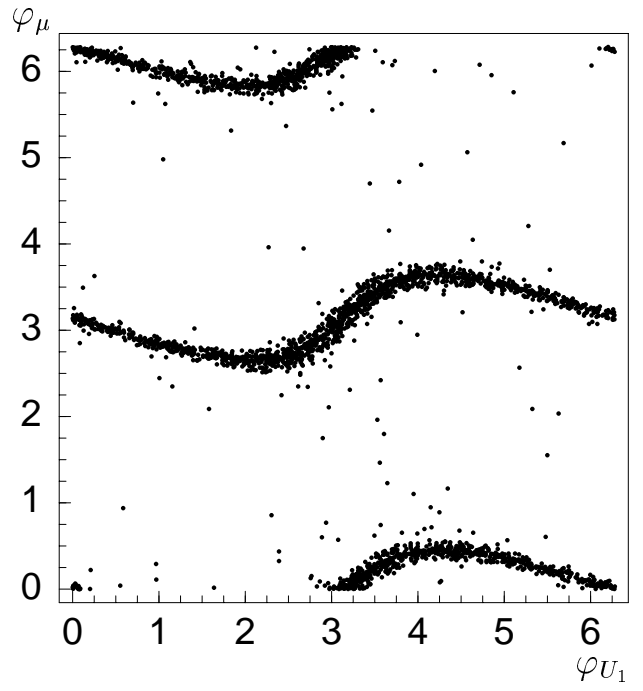

b) $10^{9} \cdot \Delta a_{\mu}$

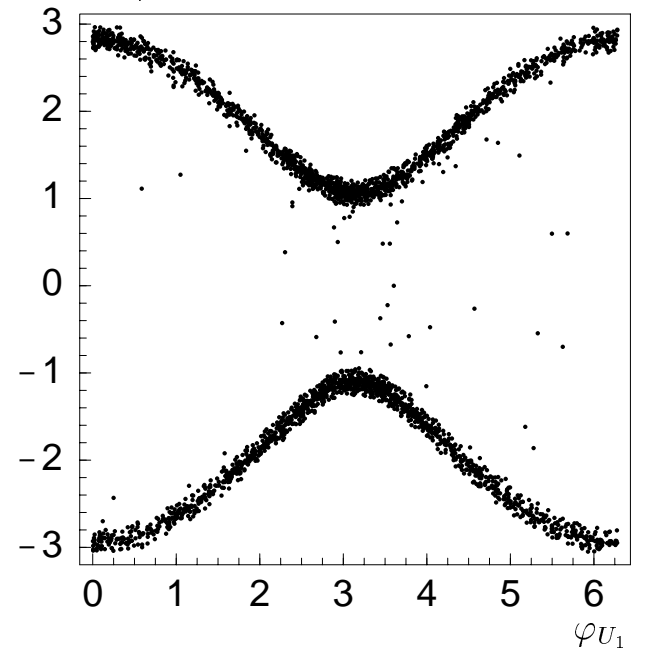

Figure 5: a) Allowed regions in the $\varphi_{\mu}-\varphi_{U_{1}}$; b) SUSY contribution $\Delta a_{\mu}$ to the anomalous magnetic moment of the muon. Here we have taken the most general form for the slepton mixings.

a) $10^{9} \cdot \Delta a_{\mu}$

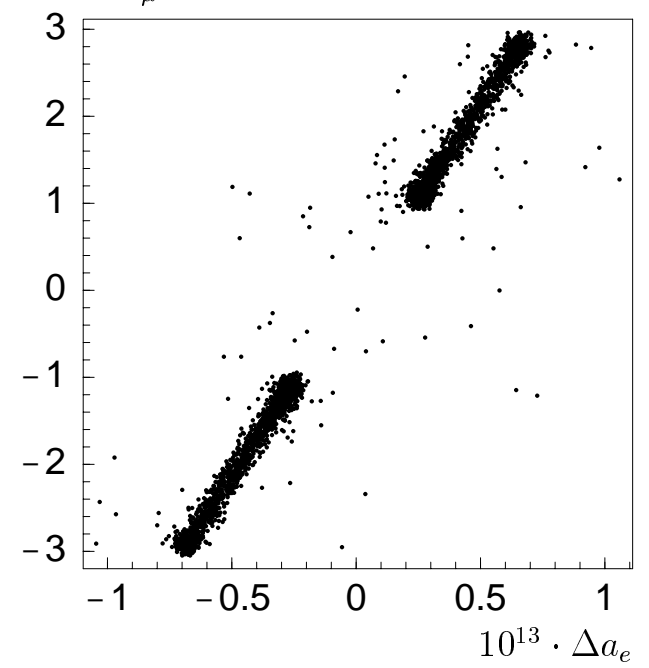

b) $10^{22} \cdot d_{\mu}$

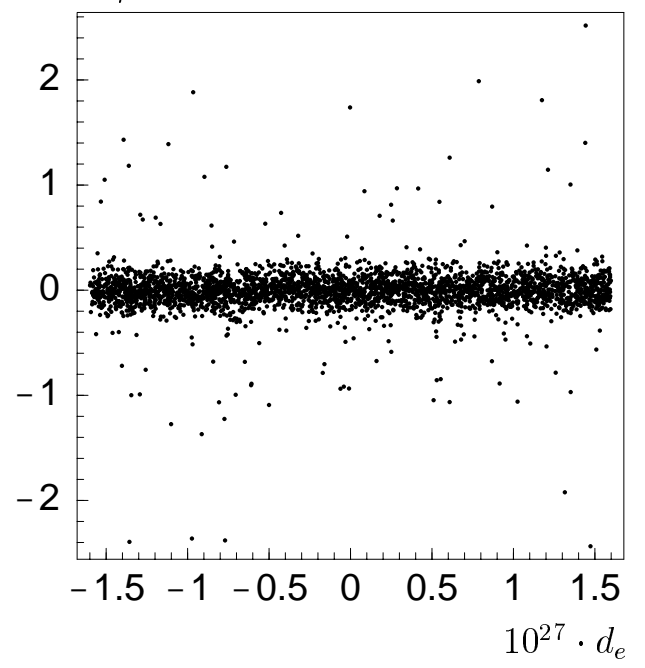

Figure 6: Correlation between a) $\Delta a_{e}$ and $\Delta a_{\mu}$ and b) $d_{e}$ and $d_{\mu}$.

would be allowed. The major differences compared to $\varphi_{\mu}=\pi / 2$ are: (i) The moduli of the $A$ parameters are smaller by about $25 \%$. (ii) There are less points with large moduli.

\section{Conclusions}

In this paper we analysed the implications on supersymmetric phases coming from the experimental restrictions on anomalous magnetic and electric dipole moments of the charged leptons and on the rare decays $\mu \rightarrow e \gamma, \tau \rightarrow e \gamma$ and $\tau \rightarrow \mu \gamma$. Here we have considered the most general mass matrices for sleptons within the MSSM, including left-right mixing, 

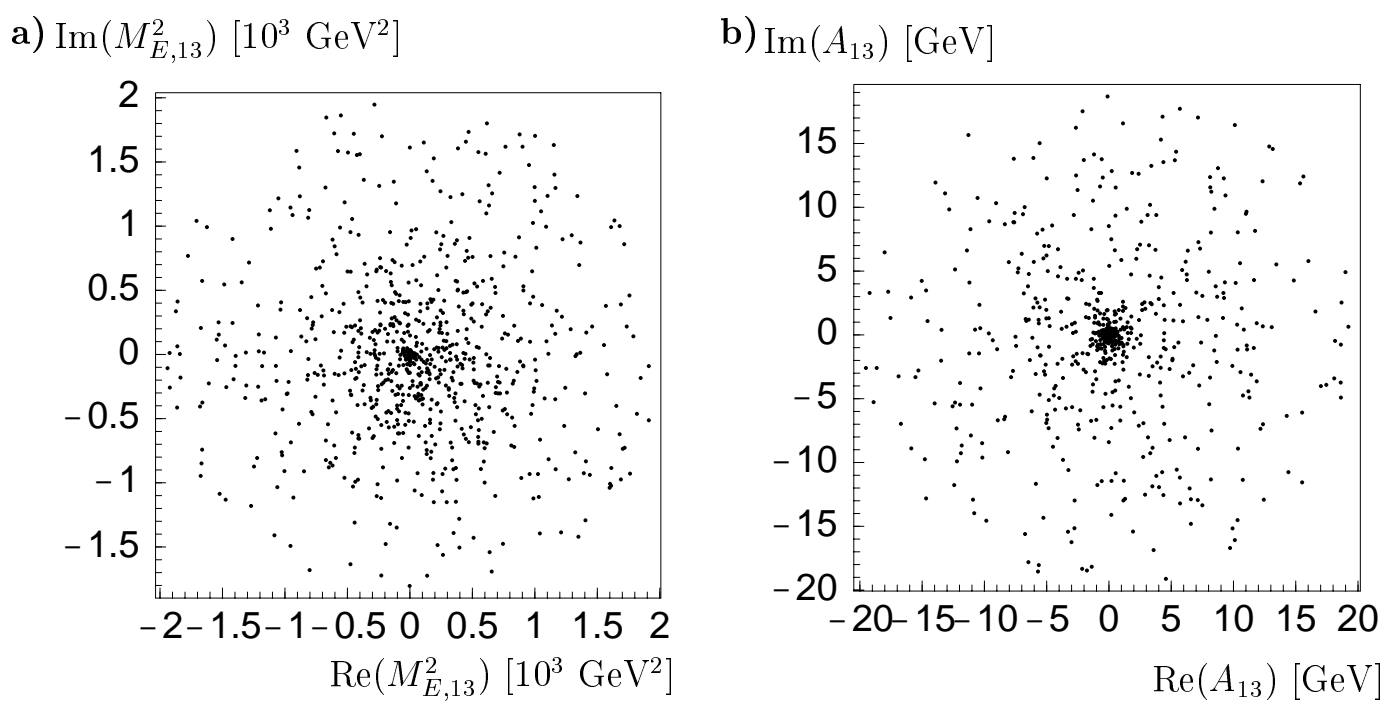

Figure 7: Real and imaginary parts of $M_{E, 13}^{2}$ and $A_{13}$ allowed by the experimental constraints. We have taken $\varphi_{\mu}=\pi / 2$ and the remaining phases have been varied as described in the the text.

flavour mixing and complex phases. For the numerical analysis we have chosen a point close to the ones discussed in the Snowmass report [20ㅁㅁ]

We have first considered two special situations, because there are many free parameters in a general scenario: (i) We have studied the case where flavour violation in the slepton sector is negligible (no off-diagonal matrix elements). We recover the known results for the phases: In general, all possible phases, especially the phase $\varphi_{\mu}$ of the $\mu$ parameter must be small (or $\pi$ ) in order to be consistent with the electric dipole moments. Only in the case that the phases of $M_{1}$ and $A_{11}$ are correlated to the phase of $\mu$, the phases of $M_{1}$ and $A_{11}$ can be maximal due to cancellations of different contributions to $d_{e}$. (ii) In the case there there is only mixing between selectrons and staus, each individual contribution to $d_{e}$ due to the phases $\varphi_{\mu}, \varphi_{U_{1}}, \varphi_{M_{E, 13}^{2}}, \varphi_{M_{L, 13}^{2}}, \varphi_{A_{13}}$, and $\varphi_{A_{31}}$ is of similar magnitude. If only one of these phases is non-vanishing, it must be rather small if the slepton masses are $\mathrm{O}(100) \mathrm{GeV}$. However, if two or more phases are present, all of them including $\varphi_{\mu}$ could be large because the various contributions to $d_{e}$ may cancel each other.

In the general case with arbitrary three-generation mixing, cancellations between various flavour conserving and flavour violating contributions to $d_{e}$ are easily possible. In the numerical analysis we have obtained two main results:

(a) Significant restrictions on the allowed ranges are obtained despite the large number of unknown parameters. A good example is given in Fig. ${ }_{-i}^{5}$ From Fig. the allowed range for the new contributions to $g-2$ are limited by the two wiggly bands. Therefore, if the theoretical analysis of $g-2$ in the standard model would yield that $\Delta a$ is in the range $(1-2) \cdot 10^{-9}$ and future collider experiments would measure a similar mass spectrum as considered here, the phase $\phi_{U(1)}$ would have to be near $\pi / 2$ or $3 \pi / 2$.

Fig. $\overline{6}$ shows that the presence of lepton flavour violating phases leads to large deviations of the scaling relations such as $d_{e} / d_{\mu} \simeq m_{e} / m_{\mu}$, but to much smaller modifications 
for the scalings of $\Delta a_{e} / \Delta a_{l}$. In the case of the electron, the mass is so small that other contribution are also important and may swamp out the mass dependence almost completely. Therefore, it is possible that the EDMs of $\mu$ and $\tau$ are larger than expected from "naive" scaling.

(b) The lepton flavour violating parameters as well as $\mu, M_{1}$ and $A_{i i}(\mathrm{i}=1,2,3)$ can have large phases, despite the stringent limits on $C P$ violation. In particular, the phase of the parameter $\mu$ can be maximal even for $\mathrm{O}(100) \mathrm{GeV}$ slepton masses, in contrast to naive expectations. Therefore, the phases of the supersymmetric parameters can be as large as those in the standard model and need not be artificially small. While we have used one of the Snowmass points for our presentation of detailed numerical results, we have checked that the qualitative features of our results do not depend on this specific choice.

\section{Acknowledgments}

I am grateful to A. Bartl, W. Majerotto and D. Wyler for an interesting and fruitful collaboration. I would like to thank the organizers of the conference for providing an interesting and stimulating atmosphere. This work was supported by the Erwin Schrödinger fellowship No. J2272 of the 'Fonds zur Förderung der wissenschaftlichen Forschung' of Austria and partly by the Swiss 'Nationalfonds'.

\section{References}

[1] Y. Fukuda et al. [Super-Kamiokande Collaboration], $P h y s$. Rev. Lett. 81 $199 \overline{9})$ 1562 S. Fukuda et al. [Super-Kamiokande Collaboration], Phys.Rev. Lett. 86 (2001) 5651 r Q. R. Ahmad et al. [SNO Collaboration], 'Phys. Rev.Lett.87-2001) 071301

K. Eguchi et al. [KamLAND Collaboration], 'Ph hys. Rev. Lett. 90 (2003) 021802.

[2] K. Hagiwara et al. [Particle Data Group Collaboration], 'P hyys. Rev. D 66 $(2002) 010001$.

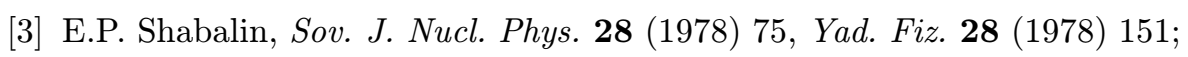
(-

A. Czarnecki and B. Krause, Acta Phys. Polon. B28 (1997) 829; ;Phys. Rev. Lett. 78 (1997)!

[4] N. V. Krasnikov, Phys. Lett. B $\mathbf{3} 8 \mathbf{8}(1996) 783$

N. Arkani-Hamed et al., Phys. Rev. Lett. 77 (1996) 1937; 'Nucl. Phys. B $\mathbf{5 0 5}(1997)$;

H. Baer et al., 'P $\bar{P} y$ s. Rev. $\mathbf{D} \mathbf{6}(2001)$

J. Hisano et al., Phys. Rev. D 60 (1999) 055000 ;

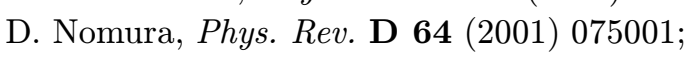

M. Guchait, J. Kalinowski and P. Roy, Eur. Phys. J. C $\mathbf{2} 1-120012163$

W. Porod and W. Majerotto, 'Phys. Rev. D $6 \mathbf{6}(2002) 015003$.

[5] R. Barbieri, L. J. Hall and A. Strumia, 'Nucl. Phys. B 445

[6] A. Romanino and A. Strumia, ${ }^{N} \bar{L} u c \bar{l}$. P $\bar{h} y s$. B $\mathbf{6} 2 \overline{2}(200 \overline{2})-73$.

[7] I. Masina and C. A. Savoy, Nucl. Phys. B 633

[8] J. L. Feng, K. T. Matchev and Y. Shadmi, 'Nucl. Phys. B 613

[9] T. F. Feng et al.,Phys. Rev. D 68 8 $(2003) 016004$ 


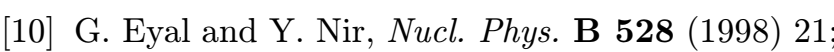

S. Abel, S. Khalil and O. Lebedev, 'Nucl. Phys. B 606 (2001) 151.

[11] M. Fukugita and T. Yanagida, Phys. Lett. B 174 (1986) 45i;

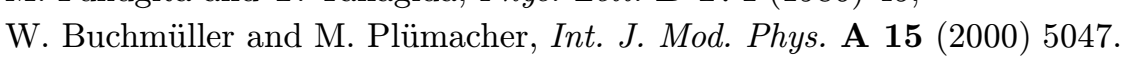

[12] See e.g. L.R. Hunter, Talk at the workshop Tests of Fundamental Symmetries in Atoms and Molecules, Harvard, 2001, http://itamp.harvard.edu/fundamentalworkshop.html.

[13] Y. K. Semertzidis et al., 'hep-ph/0012087.

[14] T. Mori et al., R-99-05, http://meg.web.psi.ch

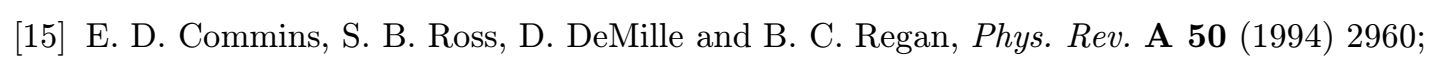
B. C. Regan,E. D. Commins, C.J. Schmidt and D. DeMille, $P$ hys. Rev. Lett. 88 $\mathbf{8}(2002)$ (- - - - - - - -

[16] J. Bailey et al. [CERN-Mainz-Daresbury Collaboration], 'Nucl. Phys. B 150 (1979) 1'.

[17] E. P. Sichtermann [the Muon g-2 Collaboration], hhep-ex/0301003.

[18] F. Jegerlehner, IJ. Phys.

M. Davier, S. Eidelman, A. Höcker and Z. Zhang, 'Eur. Phys. J.

[19] A. Bartl, W. Majerotto, W. Porod and D. Wyler, Phys.Rev. D68 20032053005

[20] B. C. Allanach et al., 'Eur. Phys. J. C25 2002$) 113$ '

[21] T. Ibrahim and P. Nath, 'Phys.Rev. D 58 (1998) 111301; Phys. Rev. D64 20012093002

T. Falk and K. Olive, Phys. Lett. B 439 (1998) 71;

U. Chattopadhyay, T. Ibrahim and P. Roy, iPhys. Rev. $\mathbf{D} \mathbf{6} \overline{4}(\overline{2001}) \overline{0} \overline{3} 0 \overline{0} \overline{;}$

V. Barger et al., 'Phys. Rev. D 64.

[22] A. Bartl et al., Phys. Rev. D.60 (1999) 073003:

[23] M. Brhlik, G. J. Good and G. L. Kane, 'Phys. Rev. D $5 \overline{9}(\overline{1} \overline{9} 9 \overline{9}) 1150 \overline{4} \overline{4}$

M. Brhlik, L. L. Everett, G. L. Kane and J. Lykken, 'Phys.Rev. Lett. 83 (1999) 2124 , 'Phys.' Rev. D $6 \mathbf{2}(2000)-035005$

[24] J.L. Feng and K.T. Matchev, Phys. Rev. Lett. 86 (2001) 3480;

L. Everett, G.L. Kane, S. Rigolin and L. Wang, 'Phys. Rev. Lett. $86-\overline{6}(20 \overline{1}) \overline{3} \overline{4} 8 \overline{4}$

T. Ibrahim, U. Chattopadhyay and P. Nath, Phys.Rev. D $64(20012016010 ;$

J. Ellis, D.V. Nanopoulos and K.A. Olive, 'Phys. Lett. B $\mathbf{5 0 8}(2001) 65_{0}^{\prime}$

S. Komine, T. Moroi and M. Yamaguchi, 'Phys. Lett. B 507 (2001) 224;

A. Bartl et al., Phys. Rev. $\mathbf{6 4}(20012076009$.

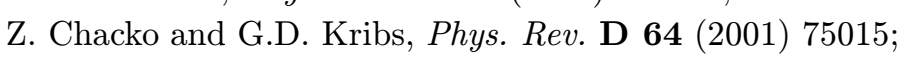

D.G. Cerdeno et al., Phys. Rev. D $\mathbf{6} \mathbf{4}(2001) 093012$;

U. Chattopadhyay and P. Nath, Phys. Rev. $\mathbf{6} \mathbf{6}(2002) 093001$;

S.P. Martin and J.D. Wells, Phys.Rev. D $\mathbf{6} 7(2003) 015002$ 